\title{
Recent Developments in Hyperspectral Imaging for Assessment of Food Quality and Safety
}

\section{Hui Huang, Li Liu and Michael O. Ngadi*}

Department of Bioresource Engineering, McGill University, Macdonald Campus, 21,111 Lakeshore Road, Ste-Anne-de-Bellevue, QC H9X 3V9, Canada;

E-Mails: hui.huang3@mail.mcgill.ca (H.H.); li.liu5@mcgill.ca (L.L.)

* Author to whom correspondence should be addressed; E-Mail: michael.ngadi@ mcgill.ca; Tel.: +1-514-398-7779; Fax: +1-514-398-8387.

Received: 25 September 2013; in revised form: 7 April 2014 / Accepted: 8 April 2014 /

Published: 22 April 2014

\begin{abstract}
Hyperspectral imaging which combines imaging and spectroscopic technology is rapidly gaining ground as a non-destructive, real-time detection tool for food quality and safety assessment. Hyperspectral imaging could be used to simultaneously obtain large amounts of spatial and spectral information on the objects being studied. This paper provides a comprehensive review on the recent development of hyperspectral imaging applications in food and food products. The potential and future work of hyperspectral imaging for food quality and safety control is also discussed.
\end{abstract}

Keywords: hyperspectral imaging; image processing; food quality and safety

\section{Introduction}

With the current growing need for low production costs and high efficiency, the food industry is faced with a number of challenges, including maintenance of high-quality standards and assurance of food safety while avoiding liability issues. Meeting these challenges has become crucial in regards to grading food products for different markets. Food companies and suppliers need efficient, low-cost, and non-invasive quality and safety inspection technologies to enable them to satisfy different markets' needs, thereby raising their competitiveness and expanding their market share.

Quality and safety of food are usually defined by physical attributes (e.g., texture, color, marbling, tenderness), chemical attributes (e.g., fat content, moisture, protein content, $\mathrm{pH}$, drip loss), and 
biological attributes (e.g., total bacterial count). Traditionally, assessment of quality and safety involves human visual inspection, in addition to chemical or biological determination experiments which are tedious, time-consuming, destructive, and sometimes environmentally unfriendly. These necessitate the need for accurate, fast, real-time and non chemical detection technologies, in order to optimize quality and assure safety of food.

With recent advancements in computer technology and instrumentation engineering, there have been significant advancements in techniques for assessment of food quality and safety. Machine vision and NIR spectroscopy are two of the more extensively applied methods for food quality and safety assessment. Machine vision techniques based on red-green-blue (RGB) color vision systems have been successfully applied to evaluate the external characteristics of foods [1-6]. Normal machine vision systems are not able to capture broad spectral information which is related to internal characteristics, hence computer vision has limited ability to conduct quantitative analysis of chemical components in food. Spectroscopy is a popular analytical method for quantification of the chemical components of food. The tight relationship between NIR spectra and food components makes NIR spectroscopy more attractive than the other spectroscopic techniques. However these spectral methods were proved inefficient when it comes to heterogeneous materials such as meat, owing to the fact that they are not capable of obtaining any spatial information about objects [7-10]. To solve the problem, repeated detection or ground of objects were recommended, which would raise the error or make the techniques destructive.

Due to the limitations of regular machine vision and spectroscopic techniques, hyperspectral imaging was developed. Hyperspectral imaging was originally developed for remote sensing applications [11]. It can be used to obtain spectral and spatial information of an object over the ultraviolet, visible, and near-infrared spectral regions (300 nm-2,600 nm) [12]. According to Gowen et al. [13], hyperspectral imaging has several merits over RGB imaging, NIR spectroscopy and multispectral imaging, including the ability to collect large and detailed spectral and spatial information. Because of the inherent merits of this technique, it has been put to application in a number of fields including agriculture [14,15], pharmaceutical [16,17], and material science [18]. Applications of hyperspectral imaging in food quality and safety include detection of contaminations [19,20], identification of defects [21,22] and quantification of constituents [23]. Recently, the technique has become more and more popular in food quality control in order to meet consumer demands and the challenge of market segmentation and legal restrictions. Publications in this research area have greatly increased in number since 2008, as shown in Figure 1, which implies the strong potential of hyeprspectral imaging as a promising detection technique for food quality and safety control.

In this paper, a comprehensive review of the recent developments in hyperspectral imaging systems and applications in food and food products is provided. Compared to other recently published review articles [24-27] which focused on the applications of hyperspectral imaging in food quality inspection, this paper highlights the optical fundamentals of hyperspectral imaging and the most recent advances in the configurations and applications of hyperspectral imaging in food quality and safety control. 
Figure 1. The number of publications about hyperspectral imaging applications in food.

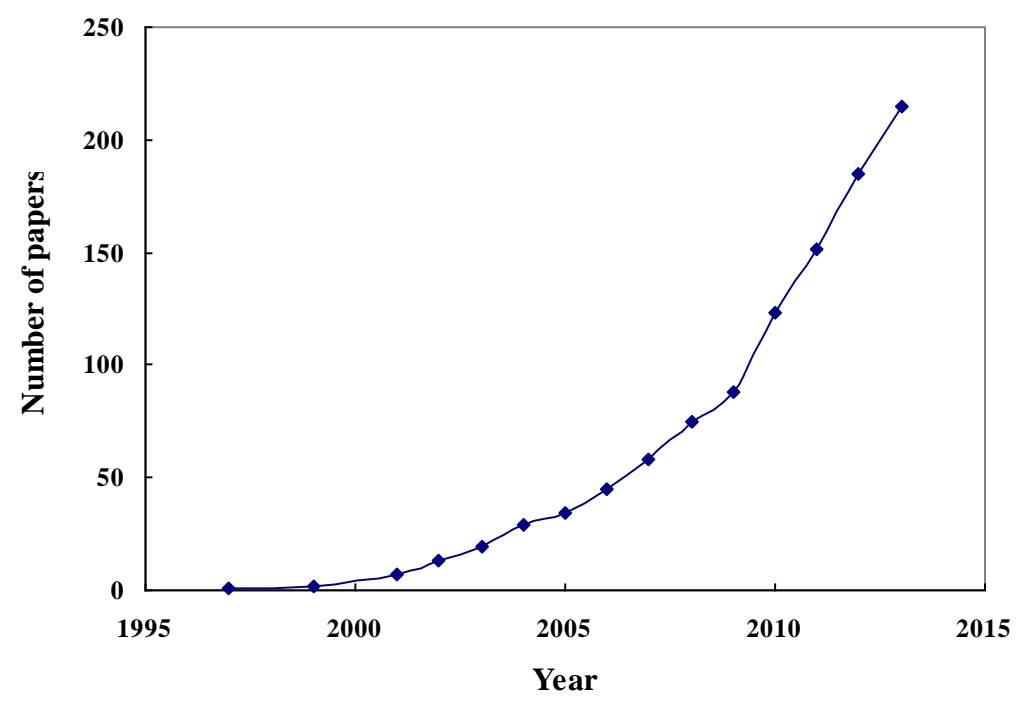

\section{Hyperspectral Imaging}

\subsection{Optical Fundamentals of Hyperspectral Imaging}

At the molecular level, all food samples continuously emit and absorb energy by lowering or raising their molecular energy levels. The wavelengths at which molecules absorb, reflect, and transmit electromagnetic radiation are characteristics of their structure [28]. Electromagnetic waves usually include ultraviolet radiation (UV), visible light (VIS), NIR, mid-infrared, and far-infrared (FIR). Each region is related to a specific kind of atomic or molecular transition corresponding to different frequencies. As with any biological material, food tissues are held together by several different molecular bonds and forces. Water, carbohydrates and fats are rich in $\mathrm{O}-\mathrm{H}$ or $\mathrm{C}-\mathrm{H}$ bonds. Organic compounds and petroleum derivatives are rich in $\mathrm{C}-\mathrm{H}$ or N-H bonds. When a food sample is exposed to light, electromagnetic waves are transmitted through it, the energy of incident electromagnetic wave changes because of the stretching and bending vibrations of chemical bonds such as $\mathrm{O}-\mathrm{H}, \mathrm{N}-\mathrm{H}$ and $\mathrm{C}-\mathrm{H}$. This makes spectroscopy able to provide characteristic and detailed fingerprints of food samples by using these observed changes in molecular energy levels.

At the macro level, the electromagnetic wave is observed as light, and the transitioning of the incident electromagnetic wave is shown as the reflection, scattering, and transmission of light. Since the absorbed part of light penetrates into the tissue of samples, the strength and wavelengths of emission and absorption depends on the physical and chemical states of the objective material. The emerging light obtained is converted to a spectrum and reshaped to images by hyperspectrometers with high signal-to-noise ratios. These obtained images, i.e., hyperspectral images, could indicate the chemical constituents and physical properties of the food samples.

\subsection{Acquisition of Hyperspectral Images}

Hyperspectral imaging systems provide hyperspectral images consisting of numerous spatial image planes of the same object at different wavelengths. The resulting hyperspectral image is achieved through the superimposition of the spatial images collected by the hyperspectral sensors, thus creating a 
three-dimensional data cube called hypercube which is then further analyzed and illustrated. These images are composed of vector pixels, and represent the composition and appearance of that particular food sample. Spectra from the data cube of different samples can be compared. Similarity between the image spectra of two samples indicates similarity of chemical composition and physical features. The hypercube usually can be constructed in three ways: area scanning, point scanning, and line scanning [13]. Due to the presence of conveyor belts (for in-line inspection) in most food processing plants, line scanning (or pushbroom) is the preferred image acquisition method. The hypercube of line scanning is acquired by composing several whole lines of an image instead of a single pixel at a time, and it is stored in the format of Band Interleaved by Line (BIL) which is a scheme for storing the actual pixel values of an image in a file band by band for each line or row of the image. The spatial and spectral information stored in BIL are analyzed simultaneously.

Hyperspectral imaging systems can be operated either in reflectance or transmittance modes. To acquire images in transmittance mode, thin sample sizes are usually used to allow light to travel through the sample. Thicker samples can be used in reflectance hyperspectral imaging measurements. Thus, food materials can be inspected as a whole in reflectance mode without the need to make slices. Examples include apples [29], cucumbers [30], mushrooms [31], and chickens [32].

Light penetration depth is defined as the depth at which the incident light was reduced by $99 \%$. It can vary according to the status, type of sample, and the detection waveband. Optical features of the light penetration depths are mainly determined by strong absorbing constituents in the sample. Research regarding the penetration depth of light in VIS and NIR range is very limited. Lammertyn et al. [33] proved that light penetration depth in apples was dependent on the detection wavelength by putting forward a non-linear model describing the correlation between the reflectance and thickness of apple slices. The penetration of apple is up to $4 \mathrm{~mm}$ in the 700-900 $\mathrm{nm}$ range and between 2 and $3 \mathrm{~mm}$ in the 900-1,900 nm range. In the research of Qin and Lu [34], the light penetration depth in tissues of apple, peach, pear, kiwifruit, tomatoes, zucchini, cucumber, and plum were calculated according to the absorption and reduced scattering spectra of the test samples at different wavelengths. The minimum light penetration depths ranged from $7.1 \mathrm{~mm}$ at $535 \mathrm{~nm}$ for the plum to $13.8 \mathrm{~mm}$ at $720 \mathrm{~nm}$ for the zucchini. The wavelengths were correlated to the absorption peaks of the major pigments in the fruits and vegetables. The maximum penetration depths ranged from $18.3 \mathrm{~mm}$ for the apple to $65.2 \mathrm{~mm}$ for the zucchini. This study highlighted that penetration depth varies a great deal depending on the type of object being studied and the applied depth. The penetration depth could have effects on the hyperspectral imaging detection. Most of the studies about penetration depth were conducted on fruits. Further research concerning penetration depth would prove beneficial by providing references for thickness determination and could be valuable for designing an appropriate and accurate sensing configuration, especially in meat products.

\subsection{Configuration of Hyperspectral Imaging System}

Typical hyperspectral imaging systems comprise hardware and software. The specific configuration may vary depending on the object to be assessed and the image acquisition technique used. Most hyperspectral imaging systems hardware platforms share common basic components (shown in Figure 2): a light source to provide illumination, usually produced by halogen-tungsten lamps; light irradiation of samples either directly or delivered by an optical fiber; a detector which obtains both spectral and spatial 
information simultaneously; a hyper-spectrograph to disperse the wavelengths of the reflected, transmitted, or scattered light and deliver signals to the photosensitive surface of the detector; an objective lens to adjust the range of light acquisition; an objective table fixed to a conveyer belt to hold and transport the sample and finally a computer to compose and store the three-dimensional hypercube.

Figure 2. Configuration of a hyperspectral imaging system.

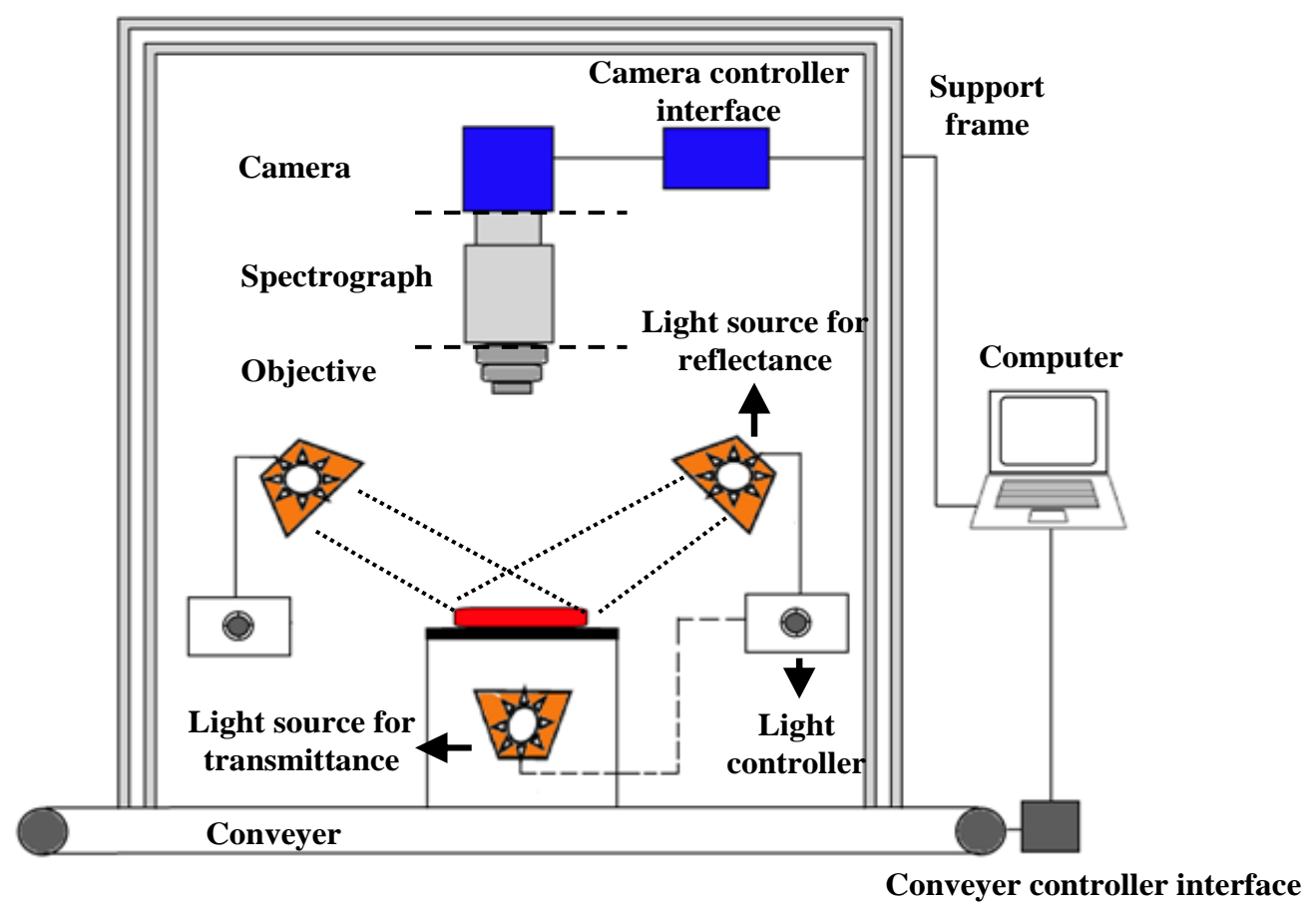

For the hyperspectral imaging system detector, there are three basic choices of cameras for this application, including silicon ( $\mathrm{Si}$ )-based charge-coupled device (CCD) or complementary metal oxide semiconductor (CMOS) cameras, indium gallium arsenide (InGaAs)-based array detectors, and mercury cadmium telluride ( $\mathrm{HgCdTe}$ )-based array detectors. The choice of the camera in a particular hyperspectral imaging system depends on the required wavelength, the quantum efficiency (QE) representing the sensitivity, and the cost. At present, the CCD camera $(300-1,100 \mathrm{~nm})$ is the most widely used VIS/NIR detector in food quality and safety analysis, with the advantage of lower cost and potential wider availability (compared to InGaAs and $\mathrm{HgCdTe}$ ). The QE of a typical Si based sensor is shown in Figure 3. The higher QE indicates higher sensitivity. The QE of Si cameras between 420 and $560 \mathrm{~nm}$ is above $50 \%$, but falls to less than $1 \%$ over $1,000 \mathrm{~nm}$. This strongly indicates that to use these sensors for imaging in the NIR region, a powerful light source is required to offer very powerful output, which can be extremely expensive and run the risk of overheating the samples during imaging.

The development of advanced instrumentation enabled the application of near infrared cameras in food processing, including the InGaAs array detector (900-1,700 nm, 1,000-2,200 nm, and 1,200-2,500 nm) and the $\mathrm{HgCdTe}$ array detector (1,000-2,600 nm). Figure 4 shows a comparison of the QE of Si-based and InGaAs-based detectors. Three types of InGaAs detectors were studied, including InGaAs1700 (900-1,700 nm), InGaAs2200 (1,000-2,200 nm), InGaAs2500 (1,200-2,500 nm). An intersection point of the QE curves of InGaAs and Si based cameras was observed around $900 \mathrm{~nm}$. The QE of InGaAs1700 falls to under $40 \%$ below $900 \mathrm{~nm}$, while the QE of Si based camera falls to $40 \%$ above $900 \mathrm{~nm}$. If the required wave band is around $900 \mathrm{~nm}$, the choice of camera may depend on whether it tends to VIS or 
NIR. In contrast of three types of InGaAs detectors, a better choice would be the InGaAs1700, whose QE is higher than $50 \%$ above $950 \mathrm{~nm}$ and keep higher than $80 \%$ between $1,000-1,600 \mathrm{~nm}$, with an average QE of $60 \%$. The InGaAs 2200 and InGaAs2500 have average QE around 50\% and 55\% separately at the desired wavelength ranges, but not as good as InGaAs1700. All types of InGaAs cameras have better sensitivity than Si-based cameras in the NIR region especially above $900 \mathrm{~nm}$. Hyperspectral imaging systems based on InGaAs camera may provide increased accuracy for assessment and analysis of food quality and safety [30,35,36], but the cost of NIR cameras is higher than for VIS/NIR cameras, which may affect the application of NIR hyperspectral imaing.

Figure 3. QE of typical Si based camera.

\section{Monochrome Quantum Efficiency}

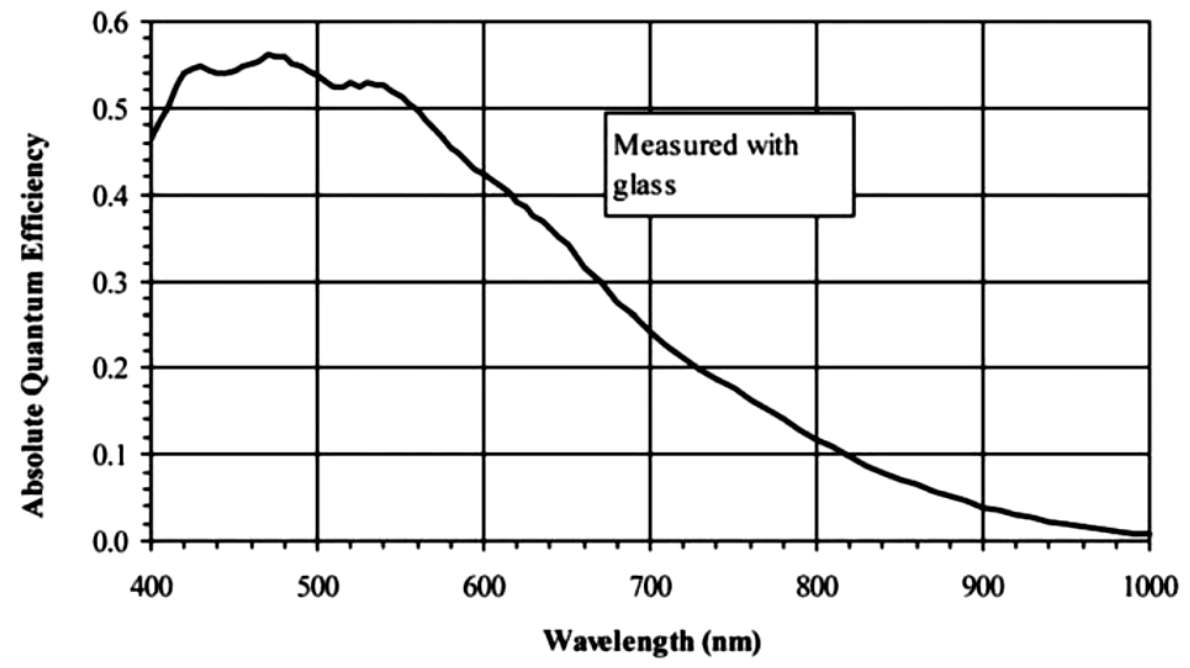

Figure 4. QE comparison of InGaAs detectors and Si-based cameras.

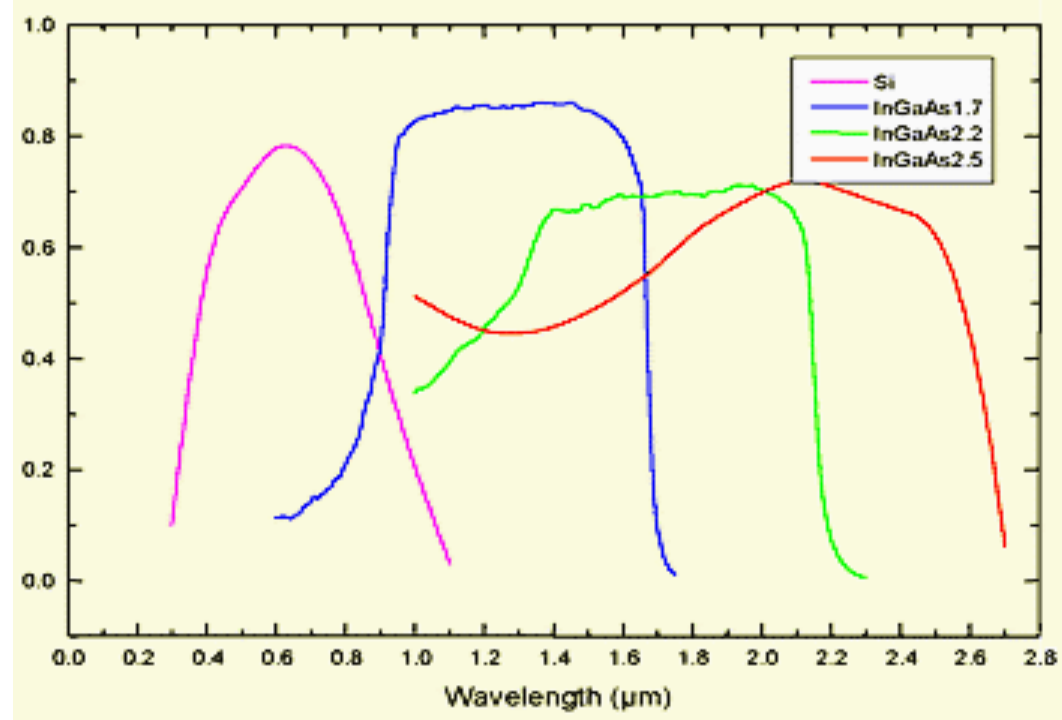

The hyperspectral imaging system and components have developed along with imaging and instrument techniques. As these technologies advance, it is expected that there will be further improvements in hyperspectral imaging systems, particularly at the higher wavelengths for more detailed analysis of food quality and safety. Very recently, a hand-held hyperspectral imaging system 
(400-720 nm) was proposed to assist in food processing facilities [37]. This system can be used to detect wear on HDPE surfaces as well as the presence of produce residues. Small amounts of juice released during the processing procedures of honeydew or cantaloupe melon can be detected using this handheld hyperspectral imaging system. This suggested that portable hyperspectral imaging equipment would be valuable in food quality and safety control.

\subsection{Spatial Resolution of Hyperspectral Imaging System}

The information that is acquired by a hyperspectral imaging system carries spatial information as well as spectral information. Spatial resolution is important for adjustment of the field of view and estimation of the scanning limit. From a practical point of view, a system with a proper spatial resolution should be selected according to the size and shape of the analyzed objects. Generally, the spatial resolution can be calculated by dividing the scanned spatial distance to the pixel numbers in each image. For point scanning system, the images are collected pixel by pixel. The spatial resolution depends on the pixel resolution of the camera. Similarly for an area scanning system, the images are collected area by area. The spatial resolutions in two spatial dimensions are the same. They are determined by the size of the detected area. For line scanning system, the dominated resolution refers to the one in the direction parallel to the slit, which is determined by several factors including zoom amount of lens, working distance, camera, imaging spectrograph, etc. [27]. In the study of Lara et al. [15], spatial resolution of $0.26 \mathrm{~mm} /$ pixel was used to study the shelf-life of spinach using a line scanning system. Kamruzzaman et al. [38] used a spatial resolution of $0.578 \mathrm{~mm} /$ pixel for visualization of minced lamb meat. Mendoza et al. [39] applied spatial resolution of $0.20 \mathrm{~mm} /$ pixel for image acquisition of apple fruit. For line scanning systems, different spatial resolutions were used in different studies and the most used one is at the millimetre level which was implied as the limitation of spatial resolution for line scanning system. It would be beneficial for food quality control if the spatial resolution of line scanning hyperspectral imaging could be reduced to the micron level.

Figure 5. Flow diagram of hyperspectral data analysis process.

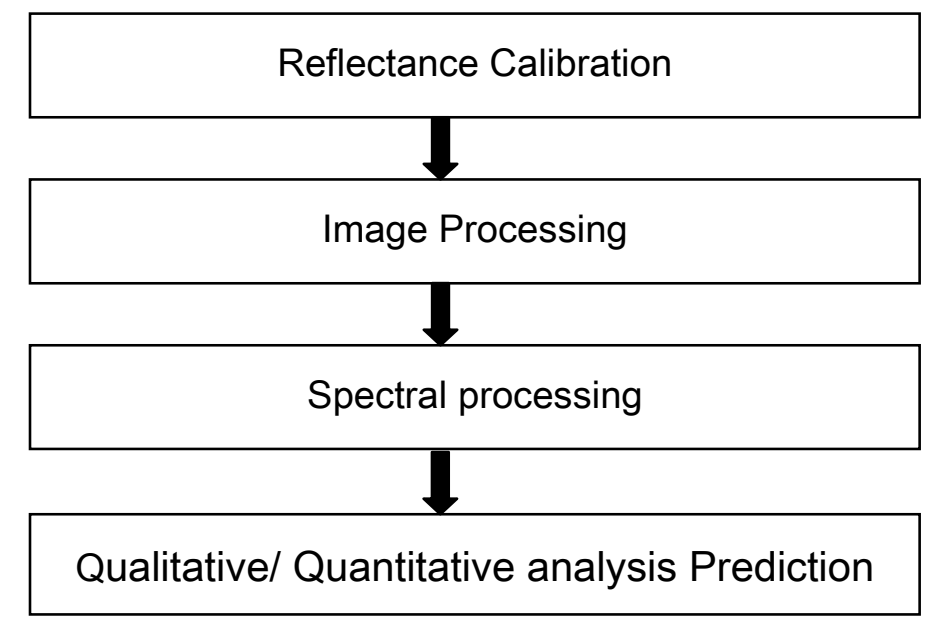




\section{Analysis of Hyperspectral Images}

The data cube produced by hyperspectral imaging systems contains a mass of information with large dimensionality. The main purpose of hyperspectral data analysis is to reduce the dimensionality and retain the useful data for discrimination or measurement analysis of food quality and safety. Corresponding to image processing technique and chemometry, many methods could be adopted to reach the detection goal. There is a main criterion that these methods should follow as Figure 5 describes, including reflectance calibration, image processing, spectral preprocessing, and qualitative analysis or quantitative analysis.

\subsection{Reflectance Calibration}

The purpose of reflectance calibration is to correct the acquired sample images from the dark current of the camera. The dark response $D$ is the background response of camera caused by dark current of the instrument. The dark response is obtained by turning off the light source, completely covering the lens with its cap, and recording the camera response. The bright response $W$ representing the total reflected light intensity from the illumination is obtained from a uniform high reflectance standard-a white ceramic tile, which reflects $99 \%$ light. After the optical reflected signal $I$ of sample is measured, the corrected reflectance value $R$ is calculated on a pixel-by-pixel basis as follows:

$$
R=\frac{I-D}{W-D}
$$

\subsection{Image Processing}

In the hyperspectral data cube, grayscale images with intensity scaling have different values in different pixels. These values are commonly used to display the compositional contrast of measured objects. In a hyperspectral data cube, the intensity of every pixel in the hyperspectral image represents different light reflectance or transmittance. After the signals are calibrated by Equation (1), several image preprocessing sequences should be carried out to provide greater contrast between distinct regions of the sample and the background. The typical image preprocessing techniques include edge detection techniques, filters, trend removals, band ratio, grey-level segmentation or thresholding techniques, digital morphology, texture, thinning and skeletonization algorithms [40], etc. Thresholding is widely used as it is necessary to segment the targeted object from the image. Region of interest (ROI), excluding redundant background in combined or original calibrated image is obtained after image preprocessing. Image preprocessing is crucial in both spectral and image processing since the selected ROI is the basis of all followed analysis.

After image preprocessing, many image analysis techniques can be used to extract useful image features for the further analysis. These include principal component analysis (PCA), minimum noise fraction, Gabor filter, wide line detector, grey level co-occurrence matrix (GLCM), variogram analysis, wavelet transform, etc. Some studies combined two or more methods to extract features more effectively [41-43].

Due to the abundance of information provided by three dimensional hyperspectral data, it is necessary to extract the important features on the sample images and to compare those feature with 
targeted features of objects. Various feature extraction methods have been developed for feature detection and extraction in hyperspectral image processing. One example is the $2 \mathrm{D}$ Gabor filter. This method involves a Gaussian function modulated by a circularly symmetric sinusoidal function or oriented harmonic function, by which the spatial frequency and directional information of image texture are embodied. The Gabor filter technique was successfully applied to extract texture features from pork [41] and egg images [43] for pork quality classification and early embryo development detection, respectively. Figure 6 demonstrates an example of using Gabor filters to extract the texture feature from hyperspectral images of a pork image. ROI of pork was preliminary selected and applied to remove the useless information from original pork image. Gabor filters were performed on the hyperspectral images of the tested pork to extract the textural feature and obtain the filtered image. Finally, averaging was applied to images at all wavelengths to calculate the filtered mean spectra (shown as a plot). Useful information could be obtained by Gabor filters, which however, does not exclude redundant information. Hence, PCA, which is a technique that reduces the redundant features, was applied in the study of Liu et al. [41]. The principle of PCA was explained by Qin et al. [27]. PCA has been widely used to reduce dimension, compress data, extract feature, and even identify key wavelengths in the application of hyperspectral imaging for food quality and safety control [38,42,44-52].

Another method namely wide line detector was applied to extract the line feature from red-green-blue images of pork. The red, green, and blue images were acquired using a hyperspectral imaging system. Figure 7 shows examples of marbling extraction results, where lines with different width were efficiently extracted by wide line detector. According to Liu et al. [53], the wide line detector is insensitive to contrast between the object and surrounding pixels. Therefore, wide line detector is able to extract either narrow or wide lines from an image. The study is a good example of the application of pattern recognition techniques for hyperspectral analysis.

Figure 6. Gabor filter for extracting texture features from a ROI of a pork image. (a) Selected ROI of pork, (b) applied Gabor filter, (c) Gabor filtered image, (d) extracted texture features.

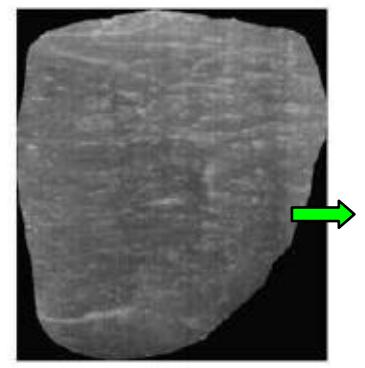

(a)

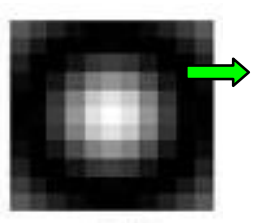

(b)

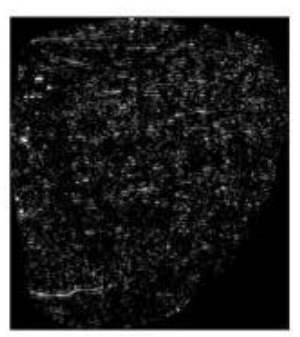

(c)

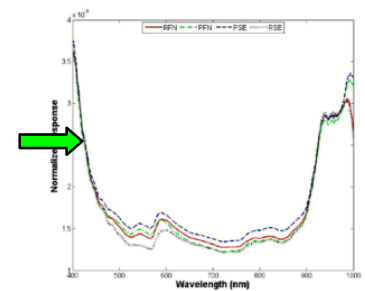

(d) 
Figure 7. Wide line detector for extracting line feature from red, green, and blue images of pork.

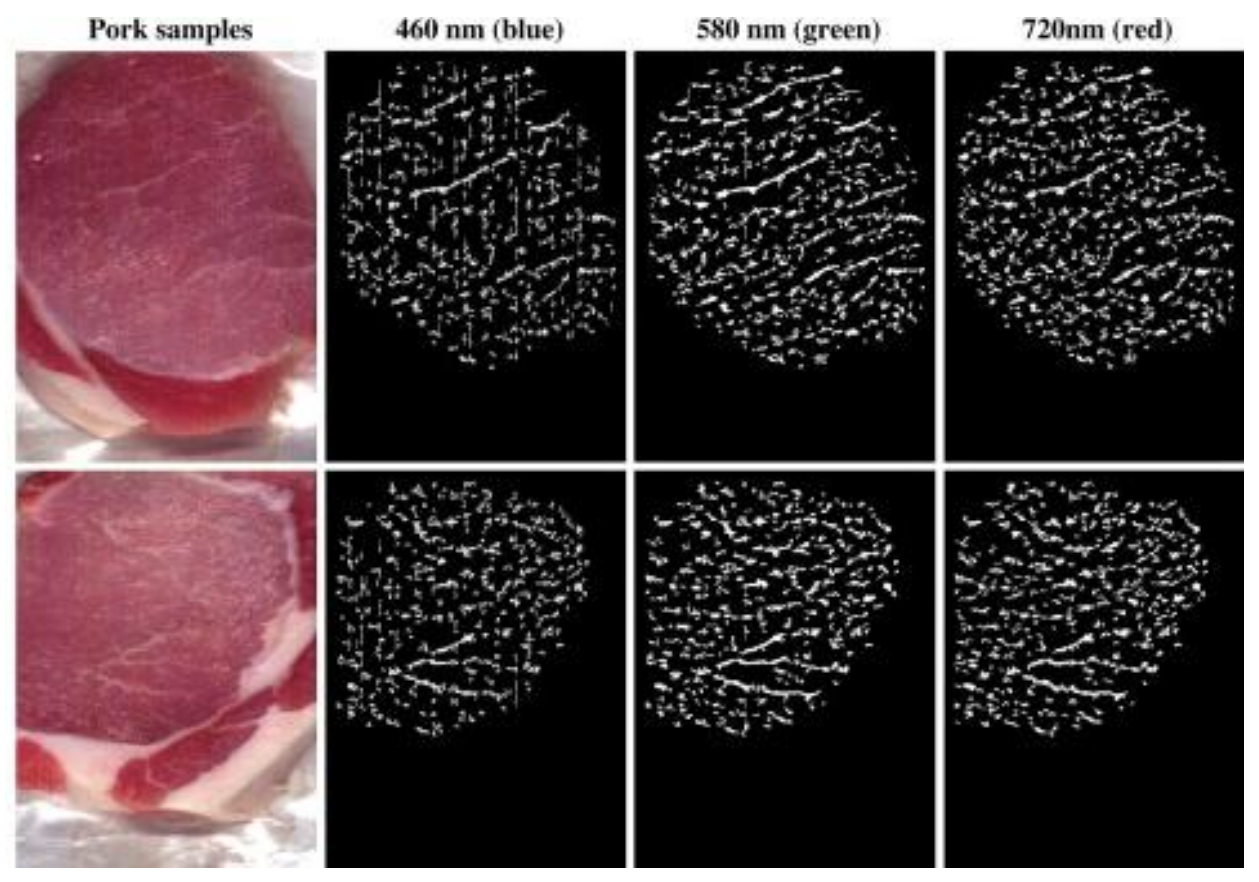

\subsection{Spectral Processing}

The basic spectral processing is spectral averaging. A mean spectrum is calculated by averaging the value of pixels included in the ROI. Individual output, i.e., an individual spectrum, corresponds to individual samples. Interference signal (baseline drift, particle deviation, surface heterogeneity) could exist in spectrum. Therefore, spectral preprocessing techniques should be used to remove these nonchemical biases from the spectral information. There are a number of preprocessing techniques in spectral processing, including Savitzky-Golay filter, multiple scattering correction (MSC) for particle scattering effect elimination, first or second derivative, smoothing, and standard normal variate (SNV) [54]. Signal to noise ratio could be strengthened and more effective signals could be distinguished by computing correction factors using different preprocessing techniques. Recently, a preprocessing method named Modified Lorentzian Distribution (MLD) function, which was proposed by Peng and Lu [55], was applied to create a curve fit for beef spectral information [29], by which the effective scattering information of beef tenderness and $\mathrm{pH}$ value was magnified and the prediction result was improved effectively.

\subsection{Qualitative Analysis and Quantitative Analysis}

Application of hyperspectral imaging in practice may be limited due to the resulting large and computationally excessive hypercube. Thus, it is necessary to extract the characteristic wavelength by operating qualitative or quantitative analysis. These analyses aim to identify or explore the relationship between food features and spectral characteristics. Another important part of spectral processing is the optimization of wavelengths. For qualitative analysis, discriminate analytical tools such as manual observation, principle component analysis (PCA), linear discriminant analysis (LDA), and $k$-means clustering are usually employed to classify or evaluate samples according to a selection criterion [26,56]. 
Recently, Ariana and Lu [30] applied a hybrid approach, namely partial least squares discriminant analysis (PLSDA), which employs both partial least squares regression (PLSR) and LDA, to extract hyperspectral feature of defective cucumber and pickles $(\mathrm{R}>0.80)$. In this study, $\mathrm{K}$-nearest neighbor (KNN) was also applied to compare with PLSDA and the result indicated that PLSDA outperformed KNN. There were other reports of employment of PLSDA in image analysis of food, including pork, pickling cucumber, smoked salmon, and spinach leaves [57-60]. PLSDA was successfully practiced in those studies. However, the stability of PLSDA may be considered in further assessment [14].

Another research group [61] developed a spectral information divergence ( SID )-based classification algorithm. SID is a complex value calculated from $X$ and $Y$ :

$$
\operatorname{SID}(X, Y)=D(X \| Y)+D(Y \| X)
$$

where $X$ is a given hyperspectral pixel from the hyperspectral image; $\mathrm{Y}$ is another pixel with probability vector formed by matrix transformation from $X$ [62]. After SID quantified the spectral discrepancy by making use of the relative entropy to account for the spectral information provided by each pixel, classification algorithm was applied to SID map to distinguish normal citrus from cankered citrus.

For quantitative analysis prediction, multivariate analytical tools such as PCA, PLSR, stepwise multi-linear regression (SMLR), are usually employed for chemical content prediction [27,63]. PCA and PLSR are the mostly used modeling method. Another method, support vector machines (SVM) were applied in some studies of non-invasive food quality and safety control [14,40,64]. A research group practiced radial basis function (RBF) based on least square support vector machines (LS-SVM) in hyperspectral image processing [65]. As an improvement of support vector machines (SVM), LS-SVM was showed to have better performance. This indicated that LS-SVM is an efficient quantitative analysis tool for high dimensional data.

\section{Application of Hyperspectral Imaging in Food Analysis}

As an emerging process analytical tool, hyperspectral imaging is well suited for food quality and safety control. Rapid detection and monitoring of food quality and safety are required for online implementation in food processing system. hyperspectral imaging could be used as a powerful tool for the identification of key wavelengths in the development of online automated multispectral imaging systems. Consequently, hyperspectral imaging finds widespread use in research mainly as a tool to develop multispectral inspection equipment.

Table 1 presents a summary of typical papers published in hyperspectral detection of food quality and safety since 2008. Over the past several years, intensive research has been carried with regards to the emerging potential for hyperspectral imaging application in the food industry. As shown in Table 1, reflectance mode and VIS/NIR (400-1,000 nm) region are the major application areas, while transmittance mode and NIR $(900-1,700 \mathrm{~nm})$ region is increasingly used to monitor external or internal features of different types of raw and processed food. Although the majority of publications on hyperspectral imaging were on fruits and vegetables, there have been growing reports of work on seafood and meat. Table 1 lists the recent advances in the application of hyperspectral imaging on quality and safety analysis of different food products. 
Table 1. Summary of measurement mode, product type, analysis type, wavelength region, and modeling algorithm in representative papers published on hyperspectral imaging of food since 2008.

\begin{tabular}{|c|c|c|c|c|c|c|c|}
\hline Mode & Camera & Product & $\begin{array}{c}\text { Spectral Coverage } \\
(\mathbf{n m}) \\
\end{array}$ & $\begin{array}{c}\text { Analysis } \\
\text { Type }\end{array}$ & Image Processing & Modeling & Reference \\
\hline \multirow[t]{9}{*}{ Reflectance } & $\mathrm{CCD}$ & Almond nut & $\begin{array}{l}700-1,000 \\
950-1,390\end{array}$ & Qualitative & Not-mentioned & $\begin{array}{c}\text { Band ratio(BR), } \\
\text { Support vector machines (SVM) }\end{array}$ & [64] \\
\hline & $\mathrm{CCD}$ & Apple & $600-1,000$ & Quantitative & Thresholding $(\mathrm{TH})$ & $\begin{array}{l}\text { Partial least squares regression(PLSR), } \\
\text { Partial least squares discriminant analysis (PLSDA) }\end{array}$ & [66] \\
\hline & $\mathrm{CCD}$ & Apple & $450-1,000$ & Quantitative & Not-mentioned & Stepwise multi-linear regression(SMLR) & [29] \\
\hline & $\mathrm{CCD}$ & Apple & $400-1,000$ & Qualitative & $\mathrm{TH}$ & Artificial neural networks (ANN) & [67] \\
\hline & Not-mentioned & Apple & $600-1,000$ & Qualitative & Locally linear embedding (LLE) & SVM, PLSDA & [68] \\
\hline & EMCCD & Apple & $400-1,000$ & Quantitative & $\begin{array}{l}\text { First derivative, and } \\
\text { multi-resolution wavelet } \\
\text { transform }\end{array}$ & PLSR & [69] \\
\hline & EMCCD & Apple & $500-1,000$ & Quantitative & $\begin{array}{c}\text { First order statistics, Fourier } \\
\text { fractal texture, grey level } \\
\text { co-occurrence matrix (GLCM), } \\
\text { run length matrix (RLM), } \\
\text { directional fractal dimension } \\
\text { analysis, and multi-resolution } \\
\text { wavelet transform }\end{array}$ & 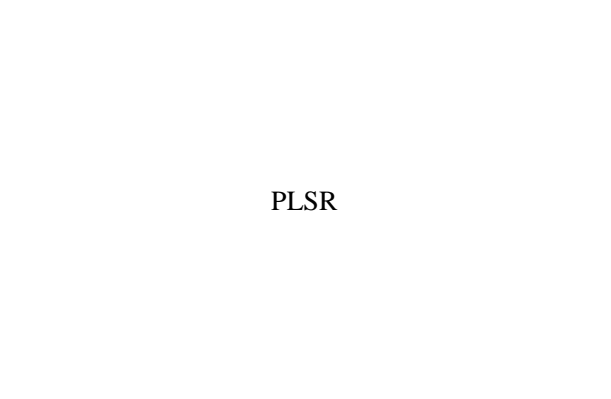 & [39] \\
\hline & Not-mentioned & Apple & $\begin{array}{l}400-1,000 \\
1,000-2,500\end{array}$ & Qualitative & $\begin{array}{l}\text { PCA, minimum noise fraction } \\
\text { (MNF) }\end{array}$ & $\begin{array}{l}\text { Soft independent modeling class analogy (SIMCA), } \\
\text { linear discriminant analysis (LDA), SVM }\end{array}$ & [42] \\
\hline & $\mathrm{CCD}$ & $\begin{array}{l}\text { Apple, peach, kiwifruit, } \\
\text { plum }\end{array}$ & $500-1,000$ & Quantitative & $\mathrm{TH}$ & Manual analysis & [34] \\
\hline
\end{tabular}


Table 1. Cont.

\begin{tabular}{|c|c|c|c|c|c|c|c|}
\hline Mode & Camera & Product & $\begin{array}{c}\text { Spectral Coverage } \\
(\mathbf{n m}) \\
\end{array}$ & $\begin{array}{c}\text { Analysis } \\
\text { Type } \\
\end{array}$ & Image Processing & Modeling & Reference \\
\hline & $\mathrm{CCD}$ & Beef & $400-1,000$ & Quantitative & $\begin{array}{c}\text { Co-occurrence matrix analysis, } \\
\text { PCA }\end{array}$ & Canonical discriminant & [44] \\
\hline & CCD & Beef & $400-1,100$ & Quantitative & MLD & Multi-linear regression (MLR) & [70] \\
\hline & $\mathrm{CCD}$ & Beef & $496-1,036$ & Quantitative & MLD & SMLR & [71] \\
\hline & $\mathrm{CCD}$ & Beef & $910-1,700$ & Quantitative & PCA & PLSR & [72] \\
\hline & $\mathrm{CCD}$ & Beef & $897-1,752$ & Quantitative & $\mathrm{TH}$ & PLSR & [73] \\
\hline & EMCCD & Blueberry & $500-1,000$ & Quantitative & $\mathrm{TH}$ & PLSR & [74] \\
\hline & $\mathrm{CCD}$ & Chicken & 389-744 & Qualitative & $\mathrm{TH}$ & BR & [32] \\
\hline & $\mathrm{CCD}$ & Chicken breast fillets & $910-1,700$ & Quantitative & $\mathrm{TH}$ & PLSR & [75] \\
\hline & $\mathrm{CCD}$ & Chicken fillets & $930-1,450$ & Quantitative & $\mathrm{TH}$ & PLSR & [76] \\
\hline & $\mathrm{CCD}$ & Citrus & $400-1,100$ & Qualitative & $\begin{array}{l}\text { Geometric factor } \\
\text { correction(GFC) }\end{array}$ & Digital elevation model (DEM) & [77] \\
\hline & EMCCD & Citrus & $450-930$ & Qualitative & $\mathrm{TH}$ & Spectral information divergence (SID) mapping & {$[61]$} \\
\hline & $\mathrm{CCD}$ & Grape seed & $914-1,715$ & Quantitative & PCA & PLSR & [49] \\
\hline & CCD & Lamb & $910-1,700$ & Qualitative & PCA & PCA & [46] \\
\hline & CCD & Lamb & $900-1,700$ & Quantitative & $\mathrm{TH}$ & PLSR & [78] \\
\hline & CCD & Minced lamb & $890-1,750$ & Quantitative & PCA & PLSR, MLR & [38] \\
\hline & CCD & Ham & $910-1,710$ & Qualitative & PCA & PCA & [47] \\
\hline & $\mathrm{CCD}$ & Dry-cured ham & $760-1,040$ & Quantitative & Not-mentioned & PLSR & [79] \\
\hline & CCD & Mandarin & $320-1,100$ & Qualitative & GFC & LDA, Classification and regression trees (CART) & [80] \\
\hline & $\mathrm{CCD}$ & Mushroom & $400-1,000$ & Quantitative & Not-mentioned & PLSR & [81] \\
\hline & $\mathrm{CCD}$ & Mushroom & $400-1,000$ & Quantitative & $\mathrm{TH}$ & PCA & [82] \\
\hline & Not-mentioned & Mushroom & $400-1,000$ & Qualitative & $\mathrm{TH}$ & PCA & [83] \\
\hline
\end{tabular}


Table 1. Cont.

\begin{tabular}{|c|c|c|c|c|c|c|c|}
\hline Mode & Camera & Product & $\begin{array}{c}\text { Spectral Coverage } \\
(\mathbf{n m}) \\
\end{array}$ & $\begin{array}{c}\text { Analysis } \\
\text { Type }\end{array}$ & Image Processing & Modeling & Reference \\
\hline & Not-mentioned & Mushroom & $450-850$ & Quantitative & Not-mentioned & MLR, Principal components regression (PCR) & [84] \\
\hline & Not-mentioned & Mushroom & $450-950$ & Qualitative & Interactive selection & PCA & [31] \\
\hline & $\mathrm{CCD}$ & Oranges & $400-1,100$ & Qualitative & PCA & PCA, BR, TH & [48] \\
\hline & $\mathrm{CCD}$ & Pork & $400-1,000$ & Quantitative & Gabor-filter, TH & PCA, K-means clustering, LDA & [41] \\
\hline & $\mathrm{CCD}$ & Pork & $400-1,100$ & Quantitative & Not-mentioned & Least square support vector machines (LS-SVM) & {$[65]$} \\
\hline & $\mathrm{CCD}$ & Pork & $460,580,720$ & Quantitative & Wide line detector & MLR & [53] \\
\hline & $\mathrm{CCD}$ & Pork & $900-1,700$ & Qualitative & PCA & PLS & [50] \\
\hline & $\mathrm{CCD}$ & Pork & $900-1,700$ & Qualitative & $\mathrm{TH}$ & PLSDA & [57] \\
\hline & Not-mentioned & Pork & $900-1,700$ & Qualitative & $\mathrm{TH}$ & PCA & [85] \\
\hline & $\mathrm{CCD}$ & Pork & $900-1,700$ & Quantitative & $\mathrm{TH}$ & PLSR & [86] \\
\hline & $\mathrm{CCD}$ & $\begin{array}{l}\text { Pickling cucumbers and } \\
\text { whole pickles }\end{array}$ & $400-740$ & Qualitative & $\mathrm{TH}$ & PLSDA, K-nearest neighbor(KNN) & [30] \\
\hline & $\mathrm{CCD}$ & Pickling cucumbers & $450-740$ & Qualitative & $\mathrm{TH}$ & PLSDA & [58] \\
\hline & CCD & Prawn & $897-1,753$ & Quantitative & $\mathrm{TH}$ & $\begin{array}{l}\text { Uninformation variable elimination, Ssuccessive } \\
\text { projections algorithm, }\end{array}$ & [87] \\
\hline & Not-mentioned & Rice seed cultivar & $874-1,734$ & Qualitative & Not-mentioned & PLSDA, SIMCA, KNN, SVM, random forest (RF) & [88] \\
\hline & $\mathrm{CCD}$ & Salmon & $400-1,100$ & $\begin{array}{l}\text { Qualitative \& } \\
\text { Quantitative }\end{array}$ & $\mathrm{TH}$ & PCA, K-means clustering, MLR & [89] \\
\hline & $\mathrm{CCD}$ & Salmon & $964-1,631$ & Quantitative & $\begin{array}{l}\text { Predictive effective } \\
\text { wavelengths (PEW) }\end{array}$ & Multiple linear regression (MLR) & [90] \\
\hline & $\mathrm{CCD}$ & Smoked salmon & $400-1,000$ & Qualitative & Quartiles segmentation, $\mathrm{TH}$ & PLSDA & [59] \\
\hline & EMCCD & Spinach leaves & $400-1,000$ & Qualitative & $\mathrm{TH}$ & $\begin{array}{l}\text { Spectral angle mapper (SAM), PLSDA, Leafy Vegetable } \\
\text { Evolution }\end{array}$ & [60] \\
\hline & $\mathrm{CCD}$ & Spinach & $400-1,000$ & Qualitative & Radiometric correction & PCA, analysis of Variance & [15] \\
\hline
\end{tabular}


Table 1. Cont.

\begin{tabular}{|c|c|c|c|c|c|c|c|}
\hline Mode & Camera & Product & $\begin{array}{c}\text { Spectral Coverage } \\
(\mathbf{n m}) \\
\end{array}$ & $\begin{array}{c}\text { Analysis } \\
\text { Type }\end{array}$ & Image Processing & Modeling & Reference \\
\hline & Not-mentioned & Wheat ears & $400-1,000$ & Qualitative & $\mathrm{TH}$ & SAM & [91] \\
\hline & FFT-CCD & Wheat & $700-1,100$ & Qualitative & PCA & $\begin{array}{l}\text { LDA, Quadratic discriminant analysis (QDA), } \\
\text { Mahalanobis discriminant classifier }\end{array}$ & [92] \\
\hline & $\mathrm{CCD}$ & Whole pickles & $400-675$ & Qualitative & $\mathrm{TH}$ & PCA & [93] \\
\hline & $\mathrm{CCD}$ & Whole grape skin & $400-1,000$ & Quantitative & Not-mentioned & PCA, Adaboost & [94] \\
\hline & InGaAs & Beef & $900-1,700$ & Quantitative & TH, BR & PLSR & [95] \\
\hline & InGaAs & Barley & $900-1,700$ & Quantitative & PCA, MNF & Maximum likelihood multinomial regression classifier & [45] \\
\hline & $\begin{array}{l}\text { InGaAs, } \\
\text { HgCdTe }\end{array}$ & Maize & $\begin{array}{l}960-1,662 \\
1,000-2,498\end{array}$ & Qualitative & $\mathrm{TH}$ & PLS-DA & [36] \\
\hline & $\mathrm{HgCdTe}$ & Maize & $1,000-2,498$ & Qualitative & PCA & PLSR & [96] \\
\hline & InGaAs & Onion & $1,000-1,600$ & Qualitative & $\mathrm{TH}$ & Manual analysis & [97] \\
\hline & InGaAs & Oat and groat & $1,006-1,650$ & Qualitative & PCA & PLS-DA & [51] \\
\hline & InGaAs & Pork & $900-1,700$ & Qualitative & Gabor filter, GLCM, TH & PLSR & [98] \\
\hline & InGaAs & Pork & $900-1,700$ & Quanlitative & Gaborfilter, GLCM, TH & PLSR & [99] \\
\hline & InGaAs & Strawberry & $1,000-1,600$ & Qualitative & $\mathrm{TH}$ & LDA & {$[100]$} \\
\hline & InGaAs & Strawberry & $1,000-1,600$ & Qualitative & Multi-band image segmentation & $\begin{array}{l}\text { Multi-band multivariate classifiers, uni-band univariate } \\
\text { classifiers, multiband decision-fusion classification }\end{array}$ & {$[101]$} \\
\hline & InGaAs & Wheat & $960-1,700$ & Qualitative & $\begin{array}{l}\text { Image cropping, feature } \\
\text { extraction }\end{array}$ & LDA, QDA, ANN & {$[102]$} \\
\hline & InGaAs & Wheat & $1,000-1,600$ & Qualitative & PCA & LDA, QDA & [103] \\
\hline
\end{tabular}


Table 1. Cont.

\begin{tabular}{|c|c|c|c|c|c|c|c|}
\hline Mode & Camera & Product & $\begin{array}{c}\text { Spectral Coverage } \\
(\mathbf{n m}) \\
\end{array}$ & $\begin{array}{c}\text { Analysis } \\
\text { Type }\end{array}$ & Image Processing & Modeling & Reference \\
\hline \multirow[t]{8}{*}{ Transmittance } & $\mathrm{CCD}$ & Cod & $448-752$ & Qualitative & $\mathrm{TH}$ & Gaussian maximum likelihood (GML) classifier & [104] \\
\hline & $\mathrm{CCD}$ & Egg & $550-899$ & Qualitative & Not-mentioned & PCA & [105] \\
\hline & InGaAs & Egg & $900-1,700$ & Qualitative & TH, Gabor filter & K-means clustering & [43] \\
\hline & CCD & $\begin{array}{l}\text { Pickling cucumbers and } \\
\text { whole pickles }\end{array}$ & $740-1,000$ & Qualitative & $\mathrm{TH}$ & PLSDA, KNN & [30] \\
\hline & $\mathrm{CCD}$ & Pickling cucumbers & $740-1,000$ & Qualitative & $\mathrm{TH}$ & PLSDA & [58] \\
\hline & CMOS & Shell-free cooked clam & $600-950$ & Qualitative & $\mathrm{TH}$ & Supervised parasite detector & [106] \\
\hline & $\mathrm{CCD}$ & Vegetable soybean & $400-1,000$ & Qualitative & Not-mentioned & Support vector data description (SVDD) classifier & [14] \\
\hline & CCD & Whole pickles & $675-1,000$ & Qualitative & $\mathrm{TH}$ & PCA & [93] \\
\hline \multirow[t]{3}{*}{ Fluorescence } & EMCCD & $\begin{array}{l}\text { Microbial biofilm } \\
\text { formation }\end{array}$ & $421-700$ & Qualitative & $\mathrm{TH}$ & PCA & [107] \\
\hline & EMCCD & Cherry tomato & $400-700$ & Qualitative & PCA & PCA & [52] \\
\hline & $\mathrm{CCD}$ & Maize & $400-700$ & Qualitative & $\mathrm{TH}$ & Discriminant analysis & [19] \\
\hline
\end{tabular}




\subsection{Fruit}

Most of the products that have been studied with hyperspectral imaging are fruits, targeting apple, citrus, pear, peach, oranges, almond nut, blueberry, citrus, grape seed, grape skin, and strawberry. The majority of these studies were carried out in reflectance mode and in the VIS-NIR range (about 400-1,100 nm). Chilling defects, diseases and quality attributes of fruits including soluble solids content, mealiness, etc., were clarified. Besides, a few studies have been carried out on strawberries and grape seeds in the NIR range (about 900-1,700 nm) [49,100,101].

One quality attribute of fruit that has been assessed using hyperspectral imaging is soluble solids content. Peng and Lu [29] designed a reflectance system to detect apple firmness and soluble solids content using stable object stage. Optic fiber and focusing lenses were used to illuminate samples as a spot light source and 2D hyperspectral images were collected. The light source used in this study was delivered by a circular beam of $1.5 \mathrm{~mm}$, which scanned the fruit $1.6 \mathrm{~mm}$ off the incident center. Ten MLD functions were proposed to fit the spectral scattering profiles and the best one was chosen as the ideal method for predicting fruit firmness and SSC using MLR. The best prediction results with two attributes of apple were got with correlation coefficient of 0.85 . In the study, over 20 wavelengths were used for prediction. This may influence the data processing speed. Later, Mendoza et al. [39] employed integrated spectral scattering and image characteristics to predict the firmness and soluble solids content of apples. The results indicated an increase of $6 \%$ of standard errors of prediction (SEP) for firmness and $3 \%$ of SEP for soluble solids content. Large latent variables were adopted in the prediction model, which indicated the necessary of a more robust prediction model for firmness and soluble solids content of apples. Leiva-Valenzuela et al. [74] used VIS/NIR hyperspectral imaging (500-1,000 nm) to determine the soluble solids content in blueberries, reaching prediction accuracies of 0.87 and 0.79 for firmness and soluble solids content, respectively.

Another quality attribute of fruit evaluated using hyperspectral imaging is mealiness. Huang and $\mathrm{Lu}$ [66] examined the relationship between reflectance hyperspectral line images and apple mealiness. The spectral scattering profiles at individual wavelengths of apples undergoing different time, images were obtained and correlated to different mealiness levels. The mealiness of the apple was determined by the hardness and juiciness. Its correlation with hyperspectral scattering profiles was predicted using PLS. Classification models with two-class or more class were built using PLSDA. The best classification accuracy was obtained in the classification of 'non mealy' and 'mealy' apples, with an accuracy of $75 \%$. This study demonstrated that hyperspectral scattering technique was potentially useful for nondestructive detection of apple mealiness and suggested that further research should focus on improving the classification accuracy especially for discrimination of less severe mealy apples. The same spatially resolved diffuse reflectance hyperspectral imaging system was used to study optical properties of fruits and vegetables including apple, pear, cucumber, tomato [34]. The study reinforced hyperspectral imaging technique's potential as a convenient attribute classification means for many fruits and vegetables. In study of Huang et al. [68], the mealiness of apple was determined using VIS/ NIR hyperspectral imaging. A classification accuracy of $82.5 \%$ was obtained using LLE algorithm-assisted SVM models.

Some work also has been done for defect detection of fruits. Qin et al. [61] examined the relationship between hyperspectral area images and citrus canker, using an Electron-Multiplying 
Charge-Coupled-Device (EMCCD) imaging device which is a type of CCD with high photosensitivity. Later, the hyperspectral area images were processed and classified to differentiate citrus canker lesion from normal and other peel diseased conditions including greasy spot, insect damage, melanose, scab, and wind scar. The analysis method was SID based classification. The overall classification accuracy was $96 \%$. It was noted that canker lesions at different developmental stages affected the SID-based classification results. Indeed, canker lesions influenced the reflectance characteristics of a given object. Further work should be targeted at the changing patterns of the citrus canker reflectance properties and incorporate canker spectra at different growth stages. Meanwhile, since this research used full spectral information which was not good for online citrus canker detection, more work could be done at exploring better method to optimize waveband combination and raise image processing speed. Further research should concentrate on improving the processing speed of the large amount of hyperspectral information.

\subsection{Vegetables}

The main application of hyperspectral imaging on vegetables includes onions, mushrooms, pickling cucumbers and whole pickles, spinach leaves, and cherry tomato. The major adopted mode is still reflectance mode, while few studies were conducted in transmittance model and fluorescence modes.

Ariana and Lu [93] developed a VIS-NIR hyperpsectral imaging system combining reflectance mode and transmission mode together, while using a moving transport platform. This system was applied to detect inner defected pickle pieces and classify pickling cucumbers and pickles, with spectral range of 500-1,000 nm. The system was capable of identifying inner defects of cucumber and pickles which were invisible to the naked eye.

Six papers have been published on mushroom quality detection using hyperspectral imaging. Taghizadeh et al. [81] investigated the shelf life (using parameters including weight loss, color, maturity index) of mushrooms under different packaging polymer films (polyvinyl chloride (PVC), polyethylene terephthalate (PET) with different levels of perforations). This research demonstrated that hyperspectral imaging has potential as an analytical tool for evaluation of shelf-life of fresh mushrooms. It also indicated hyperspectral imaging can be used to evaluate the effect of different packaging solutions especially packaging materials. It indicated that PET packaging film perforated with diameter $1 \mathrm{~mm}$ was generally superior and viable alternative to PVC film in terms of maintaining overall mushroom quality.

The bruise damage [83] and freeze damage [31] of mushroom were identified using PCA. Mushroom slice quality was measured in terms of moisture content, colour and texture $[31,83,84]$ using MLR and principal components regression (PCR). It is resulted that hyperspectral imaging is potential for damage detection and quality measurement of mushroom.

Gaston et al. [82] concerned with the prediction of polyphenol oxidase (PPO) activity on mushroom. PCA was used as a data analysis method. The result of this study revealed the possibility of developing a sensor that could rapidly identify mushrooms with a higher likelihood to develop enzymatic browning. Indeed, this study highlights the utility of hyperspectral imaging in terms of safety and quality management in the food industry. 


\subsection{Meat}

Most meat researches related to hyperspectral detection were performed on pork, beef, and chicken fillet. Lamb [46] and ham [47,79] were also investigated. Liu et al. [41] applied a Gabor filter which is used in pattern recognition to preprocess hyperspectral images of pork. PCA was used to compress spectral features over the entire wavelengths (400-1,000 nm) into principal components (PCs). 'Hybrid' PCs were created by combining PCs from hyperspectral images with PC(s) from Gabor-filtered images. Both K-means clustering and LDA were applied to classify pork samples. The overall unbiased statistical classification accuracy reached $84 \pm 1 \%$. The comparison results of hyperspectral images and Gabor-filtered images based analysis proved that the texture features extracted by Gabor filter offered useful information for the differentiation of different levels of pork quality.

Liu et al. [53] proposed an automatic and objective evaluation method for pork marbling score assessment in the view of pattern recognition. The wide line detector which is adopted in pattern recognition was applied for marbling extraction. Standard charts of marbling scores were used. The seven levels of marbling score were classified with an accuracy of 99\%. The data used by Qiao et al. [23] were employed in this study to investigate marbling score estimation. The assessment result was much more accurate than Qiao's result in which the texture indices were extracted from the hyperspectral images by co-occurrence matrix. This study alleviated the contrast problem brought about by PSE and PFN samples when subjective marbling assessment was made on these pale and reflective pork samples. Huang et al. [99] studied the wide line detector and Gabor filter for NIR spectral image analysis of pork marbling. It turned out that the Gabor filter outperformed the wide line detector for processing of NIR images. Huang et al. [98] applied a Gabor filter and GLCM to determine the intramuscular fat content in fresh loin cut. The best results of correlation coefficients of calibration and cross validation ( 0.89 for both) were obtained for non-destructive prediction of IMF content of intact pork using the Gabor filter. The results of these studies implied that hyperspectral imaging has great potential to predict the fat attributes of pork. Proper image processing technique could improve the accuracy of estimation.

Peng et al. [65,70] studied the bacterial spoilage process in beef and pork, respectively using total NIR/VIS reflectance hyperspectral imaging system $(400-1,000 \mathrm{~nm})$. The best prediction result (correlation coefficient $=0.95$, standard error of prediction $(\mathrm{SEP})=0.30$ ) was obtained using combination of scattering parameters. These two studies demonstrated the great potential of hyperspectral imaging in bacterial activity which causes quality change of food. Multi-linear regression (MLR) using SMLR selected waveband combination was preferable to separate fresh beef and unfresh beef, while LS-SVM was the preferred method used to detect pork storage time. It was shown that hyperspectral imaging can provide quantitative information for bacterial concentration in beef and pork samples. The lowest bacterial concentration in beef of the study above was $1 \times 10^{4} / \mathrm{g}$, which showed that NIR/VIS spectral signal were ideal for detection of bacteria concentration $\left(\geq 1 \times 10^{4}\right)$. The results of these two researches also noted that hyperspectral imaging could be used to predict the shelf life or the storage time of beef or pork.

Barbin et al. [85] studied the grading and classification of pork using near-infrared hyperspectral imaging (900-1,700 nm). Different to the study of Qiao et al. [23], three quality grades (PSE (pale/pinkish-gray, soft and exudative), RFN (reddish-pink, firm and non-exudative) and DFD (dark purplish red, firm and dry)) were studied as grades of pork. Obvious reflectance differences of 2nd 
derivative spectra among the pork of three quality grades were observed at wavelengths 960, 1,074, $1,124,1,147,1,207$ and 1,341 nm. Principal component analysis was carried out and the accuracy was 96\%. The results of this study indicated that pork classes could be precisely discriminated using NIR hyperspectral imaging. More work could be conducted to classify fours types (PSE, RFN, RSE (reddish-pink, soft and exudative), PFN (pale/pinkish-gray, firm and non-exudative)) of pork totally.

Different types of lamb muscles from different parts of Charollais breed were imaged and analyzed using PCA in study of Kamruzzaman et al. [46]. The results demonstrated the potential of hyperspectral imaging in quality inspection of lamb.

Another developing direction of application of hyperspectral imaging is the visualization of prediction/ distribution map of quality attributes in the tested food. Since the hyperspectral images combined spectral of each pixel and image at each wavelength together, it is convenient to generate the distribution map using the built prediction models by extracting the feature of each pixel and inputting them to the corresponding prediction model. Successful examples were demonstrated for visualization of $\mathrm{L}^{*}$ values (the lightness of the colour), $\mathrm{pH}$ values, and drip loss in pork, Enterobacteriaceae on chicken fillets, $L^{*}$ values in lamb, adulteration at different levels in pork $[38,76,78,86]$.

\subsection{Seafood}

Few studies on seafood have been reported in the last few years. The tested samples included fresh and smoked salmon, cod, prawn, and shell-free cooked clams. Considering the difficulties caused by shells, seafood holds promise as an attractive area for hyperspectral imaging research. The mostly studied object is salmon, whose fillet remains smooth and shell-free. Huang et al. [89] applied hyperspectral imaging on salmon's storage time prediction. PCA based $K$-means clustering and MLR were applied to relate hyperspectral data to the storage time and texture change of salmon, respectively. The result indicated that it is possible to predict the texture and storage time using hyperspectral imaging. Wu et al. [90] used hyperspectral imaging to measure the color distribution in salmon fillet. Successive projections algorithm was employed to select effective wavelengths. Correlation coefficients of $0.876,0.744$, and 0.803 were obtained for $\mathrm{L}^{*}, \mathrm{a}^{*}$, and $\mathrm{b}^{*}$ (three coordinates of the Lab colour space representing the lightness of the colour, its position between red/magenta and green and its position between yellow and blue), respectively. Ivorra et al. [59] studied the potential of NIR hyperspectral imaging for detection of expired vacuum-packed smoked salmon. The classification success rate of $82.7 \%$ demonstrated the potential of hyperspectral imaging as a commercial tool for identification of expired salmon.

VIS/NIR hyperspectral images were investigated by Wu et al. [87] to detect the gelatin adulteration in prawn. The combination of uninformation variable elimination and successive projections algorithm (SPA) was applied for the first time to select the optimal wavelengths in the hyperspectral image analysis. A coefficient of determination of 0.965 was obtained and gelatin in all portions of the prawn was visualized. Coelho et al. [106] used hyeprspectral imaging to detect the parasite in the shell-free cooked clam Mulinia edulis. Transmittance mode was used in this study. The range of wavelengths between 600-950 nm was identified to be sensitive, where changes were observed in the normalized optical responses of clam's mantle cavity in condition of parasite. Reduction in the normalized transmittance of clam's mantle indicated a hidden parasite inside the clam. Transmittance features over $720 \mathrm{~nm}$ achieved a 100\% detection accuracy. Normalized transmittance was suggested to be a proper 
feature for development of non-destructive parasite-detector. Further work was suggested to develop a methodology for wavelength selection in predefined conditions.

\subsection{Grains}

Hyperspectral imaging has been applied for classification of grains including maize, wheat, barley, oat and groat, soybean, and rice seed. A NIR hyperspectral imaging system (900-1,700 nm) was developed in a mathematical modeling framework to identify pregerminated barley at an early stage in order to segregate the barley kernels into low or high quality [45]. This system employed a supervised classification framework based on a set of features which are insensitive to the kernel orientation. A low classification error of $3 \%$ proved feasibility of the developed system for describing the degree of pregermination of single barley kernels. Williams et al. [36] described the application of InGaAs and HgCdTe detector-based NIR hyperspectral imaging (960-1662 nm) techniques in distinguishing between hard, intermediate and soft maize kernels. PCA and multivariate data analysis were applied to detect glassy (hard) and floury (soft) endosperm inside the maize kernels. It resulted that the InGaAs detector-based NIR hyperspectral imaging system obtained a better coefficient of determination to distinguish between glassy and floury endosperm (84.9\%) comparing to the HgCdTe detector-based NIR hyperspectral imaging system (76.3\%). Serranti et al. developed an NIR hyperspectral imaging system $(1,006-1,650 \mathrm{~nm})$ for classification of oat and groat kernels [51]. The obtained hyperspectral images were analyzed using PCA and PLS-DA to build the classification models to discriminate oat and groat kernels. Three key wavelengths, i.e., 1,132, 1,195 and 1,608 nm, were identified using a bootstrap-VIP procedure. The very high classification result (almost 100\%) strongly indicated the big potential of hyperpsectral imaging in industrial application. Another NIR hyperspectral image system (960-1,700 nm) was developed for differentiation of wheat classes grown in western Canada. Different classification models were used in the system. Classification accuracies of 94\%-100\%, 86\%-100\%, and 80\%-100\% were obtained from LDA, quadratic discriminant analysis (QDA) and ANN models, respectively. It was concluded that NIR hyperspectral imaging along with statistical and ANN classifiers has the potential to effectively classify Canadian wheat.

Some research work also has been conducted for detection of damage and contaminants of grains. Williams et al. [96] developed the NIR hyperspectral imaging system (1,000-2,498 nm) to track changes in fungal contamination of whole maize kernels. PLS regression models were established to assess the changes over time. The results indicated the possibility of the early detection of fungal contamination and activity. NIR hyperspectral imaging technology has also been applied to detect damaged wheat kernels. A NIR hyperspectral imaging system in the range of wavelengths 1,000-1,600 nm was developed for detection of insect-damaged wheat kernels [103]. LDA and QDA were employed to classify healthy and insect-damaged wheat kernels and the classification accuracy was 85\%-100\%. Later, another NIR hyperspectral imaging system $(700-1,100 \mathrm{~nm})$ was established to discriminate healthy and midge-damaged wheat kernels by the same research team [92]. Statistical features and histogram features were extracted from hyperspectral images at significant wavelengths. Three statistical classifiers were used for classification. The high average accuracy (95.3\%-99.3\%) strongly indicated the potential of NIR hyperspectral imaging for detection of damaged wheat kernels. 


\subsection{Biofilm Detection}

Recently, Jun et al. [107] reported the utilization of macro-scale fluorescence hyperspectral imaging to evaluate the potential detection of pathogenic bacterial biofilm formations on five types of food-contact surface materials: stainless steel, high density polyethylene (HDPE), plastic laminate (Formica), and two variations of polished granite. These materials are commonly used to process and handle food, and sometimes cause biofilm pollution on food surface. Spots of biofilm (E. coli O157:H7 and Salmonella biofilm) growth were produced on sample surfaces and stored and scanned by fluorescence hyperspectral imaging system using ultraviolet-A excitation (421-700 nm, including a C-mount object lens, F1.9 $35 \mathrm{~mm}$ ). PCA was used to reduce the dimensionality of hyperspectral images and an image processing method was developed based on single-band and two-band ratio techniques to select the wavebands appropriate for differentiating biofilm spots form different backgrounds. The suitable spectral fluorescence band for detecting microbial biofilm on stainless steel surfaces was $559 \mathrm{~nm}$, with overall detection rate of 95\%. For HDPE and granite, ratios between different two bands provided the most efficient results. For Formica, the results were not accurate enough to detect biofilms effectively. The result of this study showed the hyperspectral imaging could also be used to develop portable hand-held devices for sanitation inspection of food packaging, which has been a big issue for food processing. It was also noted that low cell population density may influence the accuracy of biofilm inspection of food processing surfaces. More studies could be conducted on the hyperspectral imaging biofilm detection, especially in low cell population density.

\section{Discussion and Conclusions}

Hyperspectral imaging is developing as a platform technology for food quality and safety analysis in food processing and packaging. Hyperspectral imaging could obtain the internal spectral information of samples while detecting spatial signals, which are related to the physical and chemical features of a large amount of food samples and food-contact surface materials. These signals are stored in large data cube which may slow down the data processing speed. Thus, increasing the efficiency of the identification of key wavelengths should be the center focus of upcoming studies. Improvements in the data analysis would elevate the processing speed of hyperspectral imaging data, making hyperspectral imaging more suitable for online detection, and providing the basis of multiple-spectral system production. Also, the enhancement of the sensitivity and pixel resolution of camera would help to improve the prediction accuracy of hyperspectral imaging. The achievements of the research in hyperspectral imaging strongly indicate that hyperspectral imaging, especially NIR hyperspectral imaging, has a big potential in detecting quality and safety of meat and seafood products, as well as biofilm for food packaging. More applications of hyperspectral imaging technology in food quality and safety inspection during food processing and packaging will be investigated. Future work in hyperspectral imaging could focus on issues such as higher sensitivity cameras, higher resolution systems, improvements in data processing methods, increasing detection accuracy, and expanding the range of applicable food products.

\section{Conflicts of Interest}

The authors declare no conflict of interest. 


\section{References}

1. Chao, K.; Chen, Y.R.; Early, H.; Park, B. Color image classification systems for poultry viscera inspection. Appl. Eng. Agric. 1999, 15, 363-369.

2. Lu, J.; Tan, J.; Shatadal, P.; Gerrard, D.E. Evaluation of pork color by using computer vision. Meat Sci. 2000, 56, 57-60.

3. Tan, J.L. Meat quality evaluation by computer vision. J. Food Eng. 2004, 61, 27-35.

4. O'sullivan, M.G.; Byrne, D.V.; Martens, H.; Gidskehaug, L.H.; Andersen, H.J.; Martens, M. Evaluation of pork colour: Prediction of visual sensory quality of meat from instrumental and computer vision methods of colour analysis. Meat Sci. 2003, 65, 909-918.

5. Faucitano, L.; Huff, P.; Teuscher, F.; Gariepy, C.; Wegner, J. Application of computer image analysis to measure pork marbling characteristics. Meat Sci. 2005, 69, 537-543.

6. Huang, H.; Liu, L.; Ngadi, M.O.; Gariepy, C. Prediction of pork marbling scores using pattern analysis techniques. Food Control 2013, 31, 224-229.

7. Rodbotten, R.; Nilsen, B.N.; Hildrum, K.I. Prediction of beef quality attributes from early post mortem near infrared reflectance spectra. Food Chem. 2000, 69, 427-436.

8. Prevolnik, M.; Candek-Potokar, M.; Skorjanc, D.; Velikonja-Bolta, S.; Skrlep, M.; Znidarsic, T.; Babnik, D. Predicting intramuscular fat content in pork and beef by near infrared spectroscopy. J. Near Infrared Spectrosc. 2005, 13, 77-85.

9. Barlocco, N.; Vadell, A.; Ballesteros, F.; Galietta, G.; Cozzolino, D. Predicting intramuscular fat, moisture and warner-bratzler shear force in pork muscle using near infrared reflectance spectroscopy. Anim. Sci. 2006, 82, 111-116.

10. Prieto, N.; Roehe, R.; Lavín, P.; Batten, G.; Andrés, S. Application of near infrared reflectance spectroscopy to predict meat and meat products quality: A review. Meat Sci. 2009, 83, 175-186.

11. Goetz, A.F.H.; Vane, G.; Solomon, J.E.; Rock, B.N. Imaging spectrometry for earth remote-sensing. Science 1985, 228, 1147-1153.

12. Bannon, D. Hyperspectral imaging: Cubes and slices. Nat. Photon. 2009, 3, 627.

13. Gowen, A.; O’donnell, C.; Cullen, P.; Downey, G.; Frias, J. Hyperspectral imaging-An emerging process analytical tool for food quality and safety control. Trends Food Sci. Technol. 2007, 18, 590-598.

14. Huang, M.; Wan, X.; Zhang, M.; Zhu, Q. Detection of insect-damaged vegetable soybeans using hyperspectral transmittance image. J. Food Eng. 2013, 116, 45-49.

15. Lara, M.A.; Lleó, L.; Diezma-Iglesias, B.; Roger, J.M.; Ruiz-Altisent, M. Monitoring spinach shelf-life with hyperspectral image through packaging films. J. Food Eng. 2013, 119, 353-361.

16. Gendrin, C.; Roggo, Y.; Collet, C. Content uniformity of pharmaceutical solid dosage forms by near infrared hyperspectral imaging: A feasibility study. Talanta 2007, 73, 733-741.

17. Cruz, J.; Bautista, M.; Amigo, J.M.; Blanco, M. Nir-chemical imaging study of acetylsalicylic acid in commercial tablets. Talanta 2009, 80, 473-478.

18. Garcia-Allende, P.B.; Conde, O.M.; Mirapeix, J.; Cobo, A.; Lopez-Higuera, J.M. Quality control of industrial processes by combining a hyperspectral sensor and fisher's linear discriminant analysis. Sens. Actuators B Chem. 2008, 129, 977-984. 
19. Yao, H.; Hruska, Z.; Kincaid, R.; Brown, R.L.; Bhatnagar, D.; Cleveland, T.E. Detecting maize inoculated with toxigenic and atoxigenic fungal strains with fluorescence hyperspectral imagery. Biosyst. Eng. 2013, 115, 125-135.

20. Kim, I.; Kim, M.; Chen, Y.; Kong, S. Detection of skin tumors on chicken carcasses using hyperspectral fluorescence imaging. Trans. Am. Soc. Agric. Eng. 2004, 47, 1785-1792.

21. Xing, J.; Bravo, C.; Jancsók, P.T.; Ramon, H.; de Baerdemaeker, J. Detecting bruises on 'golden delicious' apples using hyperspectral imaging with multiple wavebands. Biosyst. Eng. 2005, 90, 27-36.

22. Nagata, M.; Tallada, J.G.; Kobayashi, T. Bruise detection using nir hyperspectral imaging for strawberry (fragaria x ananassa duch.). Environ. Control Biol. 2006, 44, 133.

23. Qiao, J.; Ngadi, M.O.; Wang, N.; Gariépy, C.; Prasher, S.O. Pork quality and marbling level assessment using a hyperspectral imaging system. J. Food Eng. 2007, 83, 10-16.

24. Elmasry, G.; Barbin, D.F.; Sun, D.-W.; Allen, P. Meat quality evaluation by hyperspectral imaging technique: An overview. Crit. Rev. Food Sci. Nutr. 2012, 52, 689-711.

25. Elmasry, G.; Kamruzzaman, M.; Sun, D.-W.; Allen, P. Principles and applications of hyperspectral imaging in quality evaluation of agro-food products: A review. Crit. Rev. Food Sci. Nutr. 2012, 52, 999-1023.

26. Feng, Y.-Z.; Sun, D.-W. Application of hyperspectral imaging in food safety inspection and control: A review. Crit. Rev. Food Sci. Nutr. 2012, 52, 1039-1058.

27. Qin, J.; Chao, K.; Kim, M.S.; Lu, R.; Burks, T.F. Hyperspectral and multispectral imaging for evaluating food safety and quality. J. Food Eng. 2013, 118, 157-171.

28. Sun, D.-W. Hyperspectral Imaging for Food Quality Analysis and Control; Academic Press/Elsevier; San Diego, CA, USA, 2010.

29. Peng, Y.; Lu, R. Analysis of spatially resolved hyperspectral scattering images for assessing apple fruit firmness and soluble solids content. Postharv. Biol. Technol. 2008, 48, 52-62.

30. Ariana, D.P.; Lu, R. Hyperspectral waveband selection for internal defect detection of pickling cucumbers and whole pickles. Comput. Electron. Agric. 2010, 74, 137-144.

31. Gowen, A.A.; Taghizadeh, M.; O'donnell, C.P. Identification of mushrooms subjected to freeze damage using hyperspectral imaging. J. Food Eng. 2009, 93, 7-12.

32. Chao, K.; Yang, C.; Kim, M.; Chan, D. High throughput spectral imaging system for wholesomeness inspection of chicken. Appl. Eng. Agric. 2008, 24, 475-485.

33. Lammertyn, J.; Peirs, A.; de Baerdemaeker, J.; Nicola1, B. Light penetration properties of nir radiation in fruit with respect to non-destructive quality assessment. Postharv. Biol. Technol. 2000, $18,121-132$.

34. Qin, J.; Lu, R. Measurement of the optical properties of fruits and vegetables using spatially resolved hyperspectral diffuse reflectance imaging technique. Postharv. Biol. Technol. 2008, 49, 355-365.

35. Ariana, D.P.; Lu, R.; Guyer, D.E. Near-infrared hyperspectral reflectance imaging for detection of bruises on pickling cucumbers. Comput. Electron. Agric. 2006, 53, 60-70.

36. Williams, P.; Geladi, P.; Fox, G.; Manley, M. Maize kernel hardness classification by near infrared (nir) hyperspectral imaging and multivariate data analysis. Anal. Chim. Acta 2009, 653, 121-130. 
37. Lefcourt, A.M.; Wiederoder, M.S.; Liu, N.; Kim, M.S.; Lo, Y.M. Development of a portable hyperspectral imaging system for monitoring the efficacy of sanitation procedures in food processing facilities. J. Food Eng. 2013, 117, 59-66.

38. Kamruzzaman, M.; Sun, D.-W.; Elmasry, G.; Allen, P. Fast detection and visualization of minced lamb meat adulteration using nir hyperspectral imaging and multivariate image analysis. Talanta 2013, 103, 130-136.

39. Mendoza, F.; Lu, R.; Ariana, D.; Cen, H.; Bailey, B. Integrated spectral and image analysis of hyperspectral scattering data for prediction of apple fruit firmness and soluble solids content. Postharv. Biol. Technol. 2011, 62, 149-160.

40. Sonka, M.; Hlavac, V.; Boyle, R. Image Processing, Analysis, and Machine Vision. 2nd ed., PWS. Pub. Co.; San Francisco, CA, USA, 1998.

41. Liu, L.; Ngadi, M.; Prasher, S.; Gariépy, C. Categorization of pork quality using gabor filter-based hyperspectral imaging technology. J. Food Eng. 2010, 99, 284-293.

42. Baranowski, P.; Mazurek, W.; Wozniak, J.; Majewska, U. Detection of early bruises in apples using hyperspectral data and thermal imaging. J. Food Eng. 2012, 110, 345-355.

43. Liu, L.; Ngadi, M.O. Detecting fertility and early embryo development of chicken eggs using near-infrared hyperspectral imaging. Food Bioprocess Technol. 2013, 6, 2503-2513.

44. Naganathan, G.K.; Grimes, L.M.; Subbiah, J.; Calkins, C.R.; Samal, A.; Meyer, G.E. Visible/near-infrared hyperspectral imaging for beef tenderness prediction. Comput. Electron. Agric. 2008, 64, 225-233.

45. Arngren, M.; Hansen, P.W.; Eriksen, B.; Larsen, J.; Larsen, R. Analysis of pregerminated barley using hyperspectral image analysis. J. Agric. Food Chem. 2011, 59, 11385-11394.

46. Kamruzzaman, M.; Elmasry, G.; Sun, D.-W.; Allen, P. Application of nir hyperspectral imaging for discrimination of lamb muscles. J. Food Eng. 2011, 104, 332-340.

47. Elmasry, G.; Iqbal, A.; Sun, D.-W.; Allen, P.; Ward, P. Quality classification of cooked, sliced turkey hams using nir hyperspectral imaging system. J. Food Eng. 2011, 103, 333-344.

48. Li, J.; Rao, X.; Ying, Y. Detection of common defects on oranges using hyperspectral reflectance imaging. Comput. Electron. Agric. 2011, 78, 38-48.

49. Rodriguez-Pulido, F.J.; Barbin, D.F.; Da-Wen, S.; Gordillo, B.; Gonzalez-Miret, M.L.; Heredia, F.J. Grape seed characterization by nir hyperspectral imaging. Postharv. Biol. Technol. 2013, 76, 74-82.

50. Barbin, D.F.; Elmasry, G.; Sun, D.-W.; Allen, P.; Morsy, N. Non-destructive assessment of microbial contamination in porcine meat using nir hyperspectral imaging. Innov. Food Sci. Emerg. Technol. 2013, 17, 180-191.

51. Serranti, S.; Cesare, D.; Marini, F.; Bonifazi, G. Classification of oat and groat kernels using nir hyperspectral imaging. Talanta 2013, 103, 276-284.

52. Byoung-Kwan, C.; Moon, S.K.; In-Suck, B.; Dae-Yong, K.; Wang-Hee, L.; Jongkee, K.; Hanhong, B.; Young-Sik, K. Detection of cuticle defects on cherry tomatoes using hyperspectral fluorescence imagery. Postharv. Biol. Technol. 2013, 76, 40-49.

53. Liu, L.; Ngadi, M.; Prasher, S.; Gariépy, C. Objective determination of pork marbling scores using the wide line detector. J. Food Eng. 2012, 110, 497-504. 
54. Zeaiter, M.; Roger, J.-M.; Bellon-Maurel, V. Robustness of models developed by multivariate calibration. Part ii: The influence of pre-processing methods. TrAC Trends Anal. Chem. 2005, 24, 437-445.

55. Peng, Y.; Lu, R. Modeling multispectral scattering profiles for prediction of apple fruit firmness. Trans. Am. Soc. Agric. Eng. 2005, 48, 235-242.

56. Camps-Valls, G.; Bruzzone, L. Kernel-based methods for hyperspectral image classification. IEEE Trans. Geosci. Remote Sens. 2005, 43, 1351-1362.

57. Barbin, D.F.; Da-Wen, S.; Chao, S. Nir hyperspectral imaging as non-destructive evaluation tool for the recognition of fresh and frozen-thawed porcine longissimus dorsi muscles. Innov. Food Sci. Emerg. Technol. 2013, 18, 226-236.

58. Lu, R.; Ariana, D.P. Detection of fruit fly infestation in pickling cucumbers using a hyperspectral reflectance/transmittance imaging system. Postharv. Biol. Technol. 2013, 81, 44-50.

59. Ivorra, E.; Giron, J.; Sanchez, A.J.; Verdu, S.; Barat, J.M.; Grau, R. Detection of expired vacuum-packed smoked salmon based on pls-da method using hyperspectral images. J. Food Eng. 2013, 117, 342-349.

60. Diezma, B.; Lleó, L.; Roger, J.M.; Herrero-Langreo, A.; Lunadei, L.; Ruiz-Altisent, M. Examination of the quality of spinach leaves using hyperspectral imaging. Postharv. Biol. Technol. 2013, 85, 8-17.

61. Qin, J.; Burks, T.F.; Ritenour, M.A.; Bonn, W.G. Detection of citrus canker using hyperspectral reflectance imaging with spectral information divergence. J. Food Eng. 2009, 93, 183-191.

62. Chang, C.-I. An information-theoretic approach to spectral variability, similarity, and discrimination for hyperspectral image analysis. IEEE Trans. Inf. Theory 2000, 46, 1927-1932.

63. Cen, H.; He, Y. Theory and application of near infrared reflectance spectroscopy in determination of food quality. Trends Food Sci. Technol. 2007, 18, 72-83.

64. Nakariyakul, S.; Casasent, D.P. Classification of internally damaged almond nuts using hyperspectral imagery. J. Food Eng. 2011, 103, 62-67.

65. Peng, Y.; Wang, W. Prediction of pork meat total viable bacteria count using hyperspectral imaging system and support vector machines. In Proceedings of the Food Processing Automation Conference, Providence, RI, USA, 28-29 June 2008.

66. Huang, M.; Lu, R. Apple mealiness detection using hyperspectral scattering technique. Postharv. Biol. Technol. 2010, 58, 168-175.

67. Elmasry, G.; Wang, N.; Vigneault, C. Detecting chilling injury in red delicious apple using hyperspectral imaging and neural networks. Postharv. Biol. Technol. 2009, 52, 1-8.

68. Huang, M.; Zhu, Q.; Wang, B.; Lu, R. Analysis of hyperspectral scattering images using locally linear embedding algorithm for apple mealiness classification. Comput. Electron. Agric. 2012, 89, $175-181$.

69. Mendoza, F.; Lu, R.F.; Cen, H.Y. Comparison and fusion of four nondestructive sensors for predicting apple fruit firmness and soluble solids content. Postharv. Biol. Technol. 2012, 73, 89-98.

70. Peng, Y.; Zhang, J.; Wang, W.; Li, Y.; Wu, J.; Huang, H.; Gao, X.; Jiang, W. Potential prediction of the microbial spoilage of beef using spatially resolved hyperspectral scattering profiles. J. Food Eng. 2011, 102, 163-169. 
71. Cluff, K.; Naganathan, G.K.; Subbiah, J.; Lu, R.; Calkins, C.R.; Samal, A. Optical scattering in beef steak to predict tenderness using hyperspectral imaging in the vis-nir region. Sens. Instrum. Food Qual. Saf. 2008, 2, 189-196.

72. Elmasry, G.; Sun, D.-W.; Allen, P. Non-destructive determination of water-holding capacity in fresh beef by using nir hyperspectral imaging. Food Res. Int. 2011, 44, 2624-2633.

73. Elmasry, G.; Sun, D.-W.; Allen, P. Near-infrared hyperspectral imaging for predicting colour, ph and tenderness of fresh beef. J. Food Eng. 2012, 110, 127-140.

74. Leiva-Valenzuela, G.A.; Renfu, L.; Aguilera, J.M. Prediction of firmness and soluble solids content of blueberries using hyperspectral reflectance imaging. J. Food Eng. 2013, 115, 91-98.

75. Feng, Y.-Z; S Sun, D.-W. Determination of total viable count (tvc) in chicken breast fillets by near-infrared hyperspectral imaging and spectroscopic transforms. Talanta 2013, 105, 244-249.

76. Feng, Y.-Z.; Elmasry, G.; Sun, D.-W.; Scannell, A.G.M.; Walsh, D.; Morcy, N. Near-infrared hyperspectral imaging and partial least squares regression for rapid and reagentless determination of enterobacteriaceae on chicken fillets. Food Chem. 2013, 138, 1829-1836.

77. Gómez-Sanchis, J.; Moltó, E.; Camps-Valls, G.; Gómez-Chova, L.; Aleixos, N.; Blasco, J. Automatic correction of the effects of the light source on spherical objects. An application to the analysis of hyperspectral images of citrus fruits. J. Food Eng. 2008, 85, 191-200.

78. Kamruzzaman, M.; Elmasry, G.; Sun, D.-W.; Allen, P. Prediction of some quality attributes of lamb meat using near-infrared hyperspectral imaging and multivariate analysis. Anal. Chim. Acta 2012, 714, 57-67.

79. Gou, P.; Santos-Garces, E.; Hoy, M.; Wold, J.P.; Liland, K.H.; Fulladosa, E. Feasibility of nir interactance hyperspectral imaging for on-line measurement of crude composition in vacuum packed dry-cured ham slices. Meat Sci. 2013, 95, 250-255.

80. Gómez-Sanchis, J.; Gómez-Chova, L.; Aleixos, N.; Camps-Valls, G.; Montesinos-Herrero, C.; Moltó, E.; Blasco, J. Hyperspectral system for early detection of rottenness caused by penicilliumdigitatum in mandarins. J. Food Eng. 2008, 89, 80-86.

81. Taghizadeh, M.; Gowen, A.A.; O'donnell, C.P. Comparison of hyperspectral imaging with conventional rgb imaging for quality evaluation of agaricus bisporus mushrooms. Biosyst. Eng. 2011, 108, 191-194.

82. Gaston, E.; FríAs, J.S.M.; Cullen, P.J.; O'donnell, C.P.; Gowen, A.A. Prediction of polyphenol oxidase activity using visible near-infrared hyperspectral imaging on mushroom (agaricus bisporus) caps. J. Agric. Food Chem. 2010, 58, 6226-6233.

83. Gowen, A.; O’donnell, C.; Taghizadeh, M.; Cullen, P.; Frias, J.; Downey, G. Hyperspectral imaging combined with principal component analysis for bruise damage detection on white mushrooms (agaricus bisporus). J. Chemometr. 2008, 22, 259-267.

84. Gowen, A.; O’donnell, C.; Taghizadeh, M.; Gaston, E.; O'gorman, A.; Cullen, P.; Frias, J.; Esquerre, C.; Downey, G. Hyperspectral imaging for the investigation of quality deterioration in sliced mushrooms (agaricus bisporus) during storage. Sens. Instrum. Food Qual. Saf. 2008, 2, 133-143.

85. Barbin, D.; Elmasry, G.; Sun, D.-W.; Allen, P. Near-infrared hyperspectral imaging for grading and classification of pork. Meat Sci. 2012, 90, 259-268. 
86. Barbin, D.F.; Elmasry, G.; Sun, D.-W.; Allen, P. Predicting quality and sensory attributes of pork using near-infrared hyperspectral imaging. Anal. Chim. Acta 2012, 719, 30-42.

87. Wu, D.; Shi, H.; He, Y.; Yu, X.; Bao, Y. Potential of hyperspectral imaging and multivariate analysis for rapid and non-invasive detection of gelatin adulteration in prawn. J. Food Eng. 2013, $119,680-686$.

88. Kong, W.; Zhang, C.; Liu, F.; Nie, P.; He, Y. Rice seed cultivar identification using near-infrared hyperspectral imaging and multivariate data analysis. Sensors 2013, 13, 8916-8927.

89. Huang, H.; Liu, L.; Ngadi, M.O.; Simpson, B.K. Hyperspectral evaluation of changes in vacuum-packed cold-smoked salmon during storage. In Proceedings of the International CIGR Technical Symposium, Nantes, France, 18-20 April 2011.

90. Wu, D.; Sun, D.-W.; He, Y. Application of long-wave near infrared hyperspectral imaging for measurement of color distribution in salmon fillet. Innov. Food Sci. Emerg. Technol. 2012, 16, 361-372.

91. Bauriegel, E.; Giebel, A.; Herppich, W.B. Hyperspectral and chlorophyll fluorescence imaging to analyse the impact of fusarium culmorum on the photosynthetic integrity of infected wheat ears. Sensors 2011, 11, 3765-3779.

92. Singh, C.B;Jayas, D.S.; Paliwal, J.; White, N.D.G. Detection of midge-damaged wheat kernels using short-wave near-infrared hyperspectral and digital color imaging. Biosyst. Eng. 2010, 105, 380-387.

93. Ariana, D.P.; Lu, R. Evaluation of internal defect and surface color of whole pickles using hyperspectral imaging. J. Food Eng. 2010, 96, 583-590.

94. Fernandes, A.M.; Oliveira, P.; Moura, J.P.; Oliveira, A.A.; Falco, V.; Correia, M.J.; Melo-Pinto, P. Determination of anthocyanin concentration in whole grape skins using hyperspectral imaging and adaptive boosting neural networks. J. Food Eng. 2011, 105, 216-226.

95. Elmasry, G.; Sun, D.W.; Allen, P. Chemical-free assessment and mapping of major constituents in beef using hyperspectral imaging. J. Food Eng. 2013, 117, 235-246.

96. Williams, P.J.; Geladi, P.; Britz, T.J.; Manley, M. Investigation of fungal development in maize kernels using NIR hyperspectral imaging and multivariate data analysis. J. Cereal Sci. 2012, 55, 272-278.

97. Wang, W.; Thai, C.; Li, C.; Gitaitis, R.; Tollner, E.; Yoon, S.-C. Detection of sour skin diseases in vidalia sweet onions using near-Infrared hyperspectral imaging. In Proceedings of 2009 ASABE Annual International Meeting. Reno, NV, USA, 21-24 June 2009.

98. Huang, H.; Liu, L.; Ngadi, M.O.; Gariépy, C. Rapid and non-invasive quantification of intramuscular fat content of intact pork cuts. Talanta, 2014, 119, 385-395.

99. Huang, H.; Liu, L.; Ngadi, M.O.; Gariépy, C.; Prasher, S.O. Near-Infrared spectral image analysis of pork marbling based on Gabor filter and wide line detector techniques. Appl. Spectrosc. 2014, 68, 332-339.

100. Sugiyama, T.; Sugiyama, J.; Tsuta, M.; Fujita, K.; Shibata, M.; Kokawa, M.; Araki, T.; Nabetani, H.; Sagara, Y. Nir spectral imaging with discriminant analysis for detecting foreign materials among blueberries. J. Food Eng. 2010, 101, 244-252.

101. Nanyam, Y.; Choudhary, R.; Gupta, L.; Paliwal, J. A decision-fusion strategy for fruit quality inspection using hyperspectral imaging. Biosyst. Eng. 2012, 111, 118-125. 
102. Mahesh, S.; Manickavasagana, A.; Jayasa, D.S.; Paliwala, J.; Whiteb, N.D.G. Feasibility of near-infrared hyperspectral imaging to differentiate Canadian wheat classes. Biosyst. Eng. 2008, $101,50-57$.

103. Singh, C.B.; Jayas, D.S.; Paliwal, J.; White, N.D.G. Detection of insect-damaged wheat kernels using near-infrared hyperspectral imaging. J. Stored Prod. Res. 2009, 45, 151-158.

104. Sivertsen, A.H.; Heia, K.; Hindberg, K.; Godtliebsen, F. Automatic nematode detection in cod fillets (gadus morhua 1.) by hyperspectral imaging. J. Food Eng. 2012, 111, 675-681.

105. Smith, D.; Lawrence, K.; Heitschmidt, G. Fertility and embryo development of broiler hatching eggs evaluated with a hyperspectral imaging and predictive modeling system. Int. J. Poul. Sci. 2008, 7, 1001-1004.

106. Coelho, P.A.; Soto, M.E.; Torres, S.N.; Sbarbaro, D.G.; Pezoa, J.E. Hyperspectral transmittance imaging of the shell-free cooked clam mulinia edulis for parasite detection. J. Food Eng. 2013, $117,408-416$.

107. Jun, W.; Kim, M.S.; Cho, B.-K.; Millner, P.D.; Chao, K.; Chan, D.E. Microbial biofilm detection on food contact surfaces by macro-scale fluorescence imaging. J. Food Eng. 2010, 99, 314-322.

(C) 2014 by the authors; licensee MDPI, Basel, Switzerland. This article is an open access article distributed under the terms and conditions of the Creative Commons Attribution license (http://creativecommons.org/licenses/by/3.0/). 